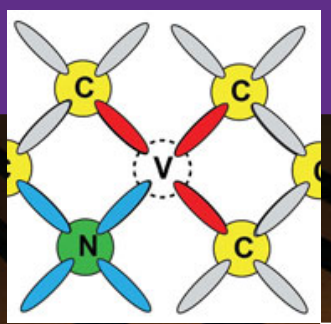

\title{
Quantum computing with defects
}

\author{
Luke Gordon, Justin R. Weber, Joel B. Varley, Anderson Janotti, \\ David D. Awschalom, and Chris G. Van de Walle
}

\begin{abstract}
The successful development of quantum computers is dependent on identifying quantum systems to function as qubits. Paramagnetic states of point defects in semiconductors or insulators have been shown to provide an effective implementation, with the nitrogen-vacancy center in diamond being a prominent example. The spin-1 ground state of this center can be initialized, manipulated, and read out at room temperature. Identifying defects with similar properties in other materials would add flexibility in device design and possibly lead to superior performance or greater functionality. A systematic search for defect-based qubits has been initiated, starting from a list of physical criteria that such centers and their hosts should satisfy. First-principles calculations of atomic and electronic structure are essential in supporting this quest: They provide a deeper understanding of defects that are already being exploited and allow efficient exploration of new materials systems and "defects by design."
\end{abstract}

\section{Introduction}

A qubit is the basic unit of information in a quantum computer. In contrast to binary "bits" that can have only two values ( 0 or 1$)$ and upon which Boolean logic is based, a qubit can be in any coherent superposition between two quantum states. ${ }^{1}$ The physical realization and control of qubits is very challenging. A range of qubit implementations is being explored, including in liquids, ${ }^{2}$ atoms,${ }^{3}$ and in solids such as superconductors ${ }^{4}$ and semiconductors. ${ }^{5}$ Loss of coherence is a major issue: In principle, the qubit should be completely isolated from unwanted external fluctuations in its environment in order to maintain its quantum state. However, a completely isolated qubit would not allow for interactions, such as entanglement, that are necessary to perform quantum manipulations and computing. Even if a single qubit can be fabricated, scalability is a major issue.

In principle, a wave function on an isolated atom would provide an intuitive, well-defined, and well-understood quantum state for use as a qubit. Unfortunately, isolated atoms do not easily lend themselves to incorporation in a quantum device; complex approaches such as ion traps or optical lattices ${ }^{3}$ are required to constrain the atoms or ions. It turns out, however, that point defects in semiconductors or insulators can display behavior that is very similar to that of isolated atoms, as illustrated in Figure 1. The electronic states associated with a vacancy, for instance, tend to have energies that lie within the forbidden bandgap, and their wave functions are constructed out of atomic orbitals on the neighboring atoms and are hence very localized — on the scale of atomic dimensions. Invoking similarities with wave functions on isolated atoms is therefore quite appropriate. The advantage of point defects is that they are firmly embedded within the host material, and decades of investigation and characterization have provided us with many tools for controlling and manipulating such defects.

Point defects tend to have a bad reputation. When unintentionally present in semiconductors, they can adversely affect the desired doping behavior and lead to degraded electronic or optical properties. The semiconductor community has therefore gone to great lengths to build a thorough understanding of such defects and to develop exquisitely precise characterization techniques, ${ }^{6-8}$ such as electron spin resonance and photoluminescence. This knowledge and toolset can now be constructively employed to design and manipulate point defects for use as qubits.

The schematic provided in Figure 1 is a qualitatively correct but highly simplified description. A serious approach to designing defects needs to be based on a rigorous quantummechanical description. For isolated atoms, we would solve the Schrödinger equation. The electronic structure of a solid, however, presents a very complicated many-body problem. Density functional theory (DFT) ${ }^{9,10}$ provides an accurate and reliable approach to tackling this issue; it has become the

\footnotetext{
Luke Gordon, Materials Department, University of California, Santa Barbara; lukegordon@engineering.ucsb.edu Justin R. Weber, Process and Materials Modeling Group, Intel Corporation; justin.r.weber@intel.com Joel B. Varley, Condensed Matter and Materials Division, Lawrence Livermore National Laboratory; varley2@|Inl.gov Anderson Janotti, Materials Department, University of California, Santa Barbara; janotti@engineering.ucsb.edu David D. Awschalom, Institute for Molecular Engineering, University of Chicago; awsch@uchicago.edu

Chris G. Van de Walle, Materials Department, University of California, Santa Barbara; vandewalle@mrl.ucsb.edu

DOI: $10.1557 / \mathrm{mrs} .2013 .206$
} 


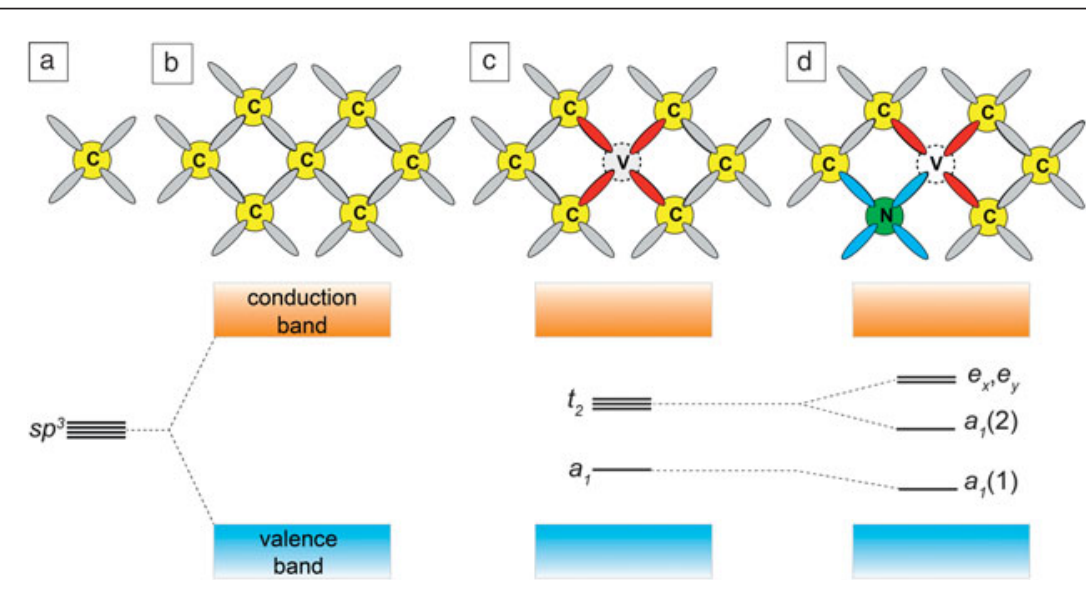

Figure 1. Schematic representation of the electronic structure of a point defect in a tetrahedrally coordinated elemental semiconductor such as diamond. (a) The electronic states corresponding to the $s p^{3}$ orbitals on an isolated $\mathrm{C}$ atom. (b) The superposition of these orbitals that gives rise to the band structure of an infinite solid. The overlap of orbitals leads to bonding and antibonding states, which broaden into valence and conduction bands. If a carbon atom is removed, as shown in (c), a vacancy is created, and the four orbitals on the surrounding atoms interact with each other in the tetrahedral environment to give rise to states with $a_{1}$ and $t_{2}$ symmetry. Because the interaction between these orbitals is weaker than the $\mathrm{C}-\mathrm{C}$ interaction that gives rise to the bands in the solid, the defect-related electronic states lie within the bandgap of the semiconductor. A symmetrylowering perturbation, such as incorporation of a nitrogen atom on one of the sites around the vacancy (d), further splits the $t_{2}$ states. Adapted from Reference 16.

standard approach for calculating structural and electronic properties of solids in general, and point defects in solids in particular. ${ }^{11}$ While DFT is, in principle, exact, practical calculations require approximations, in particular the choice of a particular functional. This functional expresses the dependence of the exchange-and-correlation potential, which encapsulates the complex many-body aspects of the problem, on the ground-state electronic charge density, which is the central quantity in DFT. The most commonly used functionals, namely the local density approximation (LDA) and the generalized gradient approximation (GGA), do a reasonable job of predicting structural properties, but fall short in the description of electronic structure. Specifically, they underestimate the bandgap of semiconductors or insulators, sometimes by more than $50 \%$. This is obviously a big problem when trying to quantitatively assess the energies of defect states within the bandgap. Hybrid functionals, which combine traditional GGA with non-local Fock exchange, have been shown to successfully overcome this problem ${ }^{12}$ and provide accurate results for bandgaps as well as for the structure, energetics, and electronic structure of defects in a wide range of materials. ${ }^{13}$

\section{The nitrogen-vacancy center in diamond}

The prototype point-defect qubit is the nitrogen-vacancy (NV) center in diamond, ${ }^{8,14}$ which consists of a nitrogen impurity next to a carbon vacancy (Figure 1d); this center can be initialized, manipulated, and read out at room temperature using optical and microwave excitations, and electric and magnetic fields. ${ }^{15}$ Figure 2 illustrates the electronic structure of this center, as calculated using hybrid DFT. ${ }^{16}$
The positioning of the defect states within the gap follows the generic pattern of Figure 1d (with the addition of spin polarization), but quantitative differences occur. For instance, the $a_{l}(1)$ states are not located within the bandgap but are resonant in the valence band. These bonding states do not play a direct role in the functionality as a spin center, and hence their energy is not directly relevant. The states that are of key importance are the spin-down $a_{l}(2)$ and $e_{x}, e_{y}$ states: Laser light of appropriate wavelength can cause a spin-conserving transition in which a spin-down electron is excited from an $a_{l}(2)$ state to an $e_{x} / e_{y}$ state; this corresponds to excitation from an ${ }^{3} \mathrm{~A}_{2}$ ground state to an ${ }^{3} \mathrm{E}$ excited state. The details of the operation of the NV center require a description in terms of such multiparticle states; this is described in great detail in a series of articles in the February 2013 issue of MRS Bulletin. ${ }^{17}$ However, the single-particle picture presented in Figure 2 is sufficient to discuss the basic features of the NV center that are key to designing similar point-defect centers. The defect states follow the pattern of Figure 1d, but spin polarization leads to different energies for spin-up and spindown states. Six electrons need to be accommodated in the defect states: Each dangling bond on a $\mathrm{C}$ atom contributes one electron, while the dangling bond on the $\mathrm{N}$ atom contributes two electrons due to the higher valence of nitrogen. In addition, an extra electron (provided by donors elsewhere in the material) is present that puts the center in a negative charge state. Filling the electronic states in order of increasing energy leads to the occupation shown in the figure, resulting in a spin-1 (triplet) state for the center.

The optical excitation energy corresponding to the transition depicted by the arrow in Figure $2 \mathrm{a}$ is evaluated by constraining the occupation to that of the excited state while keeping the atomic positions fixed to those of the ground state. The calculated energy difference $(2.27 \mathrm{eV})$ is expected to yield the peak energy of the absorption spectrum, since electronic transitions occur on a time scale much faster than atomic relaxations. If we subsequently allow the atomic positions to relax, maintaining the excited-state triplet electronic configuration, we obtain a relaxation energy of $0.25 \mathrm{eV}$ (the "Frank-Condon shift"), as illustrated in the diagram in Figure $2 \mathrm{~b}$. This configuration coordinate diagram allows the assessment of key features of optical absorption and emission processes for a point defect in which there may be significant differences in the atomic configurations of ground state and excited state. ${ }^{11}$ The energy difference between the excited state and the ground state, both with relaxed atomic configurations, corresponds to the zero-phonon line, calculated here to be $2.02 \mathrm{eV}$, and compares well with experiment, which reported a value of $1.945 \mathrm{eV}{ }^{8}$ We also obtain the peak emission energy, 
a
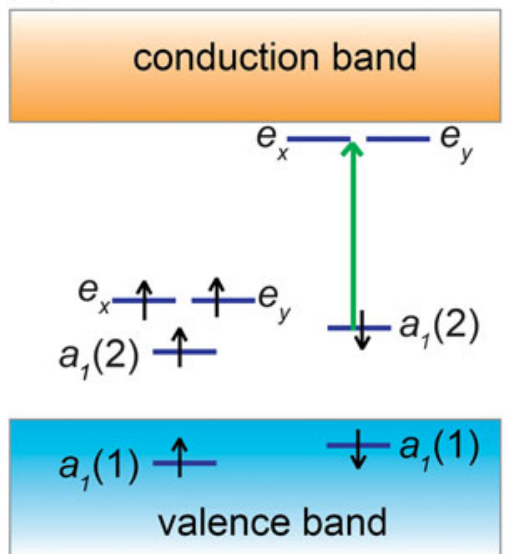

$\mathrm{b}$

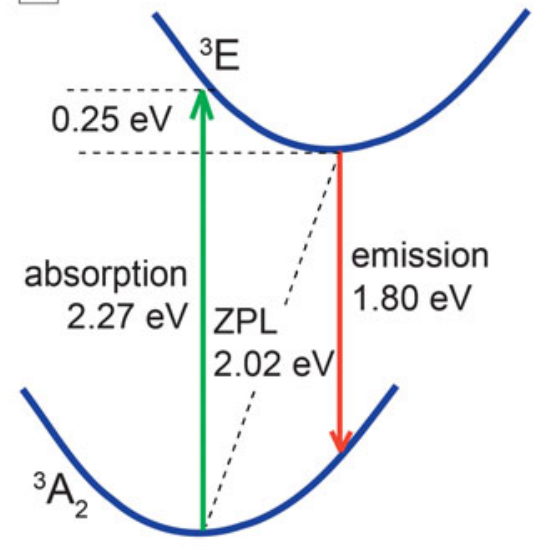

the extended states and be more delocalized. It is the energetic separation of band-to-band and defect-to-band from intra-defect optical transitions that allows the defect to be initialized and measured with high fidelity at room temperature and even well above room temperature. ${ }^{18}$ This offers significant advantages over qubit implementations that require initialization by thermal equilibration and need cryogenic temperatures to operate. The energy difference between ground and excited states must also be large to avoid thermal excitations, avoiding the destruction of spin information.

In addition to the energetic position of the defect states, the occupation of these states is also important. To function as a single-spin center, the defect must be stable in a paramagnetic ground state. As illustrated previously for the NV center (see Figure 2), this involves an exercise in electron counting: placing electrons in states of increasing energy and checking the spin state for each of those configurations. We saw that for the NV center, the desired $\mathrm{S}=1$ state required an overall negative charge state of the center; by fortunate coincidence, the Fermi level in nitrogen-doped diamond (which is determined by isolated $\mathrm{N}$ atoms acting as

at $1.80 \mathrm{eV}$, in good agreement with the experimental value of $1.76 \mathrm{eV} .^{8}$

The good agreement with experiment (to within $0.1 \mathrm{eV}$ ) is particularly impressive given that the calculations are completely $a b$ initio and involved no fitting to any experimental quantities. In addition to optical emission, a non-spin-conserving decay path also exists that includes a nonradiative transition to an intermediate spin-singlet ${ }^{1} \mathrm{~A}_{1}$ state. This transition plays a key role since it allows the center to be optically initialized into a specific spin sublevel. Recent calculations and experiments place the ${ }^{1} \mathrm{~A}_{1}$ state $1.19 \mathrm{eV}$ above the ${ }^{3} \mathrm{~A}_{2}$ ground state. ${ }^{18}$

\section{Criteria for point defects to function as qubits}

The thorough and quantitative understanding achieved for the $\mathrm{NV}$ center in diamond renders it an ideal prototype for generating a list of design criteria that other qubit candidates should satisfy. ${ }^{16}$ The highly localized nature of the bound states of the $\mathrm{NV}$ center is critical in making a robust qubit and isolating the center from sources of decoherence; indeed, coherence times of over half a second have recently been demonstrated..$^{19}$ In combination with on-chip microwave-frequency waveguides that enable quantum-control operations on sub-nanosecond time scales, ${ }^{20}$ tens of millions of coherent operations can be performed within this spin coherence time.

The localized nature of the NV-center wave functions is directly related to the position of the relevant states within the bandgap: States that are close to, or resonant with, the conduction or valence bands of the host will more strongly interact with deep donors) occurs at the right energy to put the NV center in a singly negative charge state. However, for other defects being considered as qubits, one may not be so lucky, and obtaining the correct spin state (and hence charge state) may require separate manipulation of the Fermi level through judicious incorporation of donors or acceptors. An example in the case of $\mathrm{SiC}$ is mentioned later in the text.

In addition to the ground state, the electronic structure of the defect must allow for an excited state positioned within the bandgap and accessed via a spin-conserving intra-defect optical transition. As alluded to earlier, the presence of a non-spin-conserving decay path can be important for initialization; the nature of these nonradiative transitions is still a subject of active research, which currently renders it difficult to formulate specific criteria that would ensure that such a path be present. Finally, if the qubit is to be probed by the luminescence from an excited state, the transition should be spin-conserving, and the strength of this transition should be large enough to enable efficient, high fidelity measurement of individual defect states.

As for the host material, a wide bandgap is desirable if band-to-band and defect-to-band transitions are to be avoided. Bandgaps as well as other relevant properties of some tetrahedrally coordinated hosts are listed in Table I. The host should exhibit small spin-orbit coupling in order to avoid decoherence through spin flips of the defect states. The spin-orbit splitting of the valence-band maximum can be taken as indicative of the strength of the spin-orbit interaction. Also, the constituent 
Table I. Relevant properties of potential host materials.

\begin{tabular}{|l|c|c|c|}
\hline Material & Bandgap (eV) & Spin-Orbit Splitting (meV) & Stable Spinless Nuclear Isotope \\
\hline Diamond & 5.5 & 6 & Yes \\
\hline 3C-SiC & 2.2 & 10 & Yes \\
\hline 4H-SiC & $3.2^{38}$ & 6.8 & Yes \\
\hline 6H-SiC & 2.86 & 7.1 & Yes \\
\hline AIN & 6.13 & $36^{41}$ & No \\
\hline GaN & 3.44 & 17 & No \\
\hline AIP & 2.45 & $50($ theory) & No \\
\hline GaP & 2.27 & $80(\mathrm{RT})$ & No \\
\hline AIAs & 2.15 & $275(\mathrm{RT})$ & No \\
\hline Zn0 & $3.3^{39}$ & -3.5 & Yes \\
\hline ZnS & $3.68^{40}$ & $64(\mathrm{RT})$ & Yes \\
\hline ZnSe & 2.82 & $420(\mathrm{RT})$ & Yes \\
\hline ZnTe & 2.25 & 970 & Yes \\
\hline CdS & 2.48 & 67 & Yes \\
\hline
\end{tabular}

All experimental values are from Madelung, ${ }^{21}$ unless explicitly cited otherwise. Spin-orbit splitting values are for low temperatures, except in cases where room temperature (RT) is indicated.

elements should have naturally occurring isotopes of zero nuclear spin, making it possible to eliminate spin bath effects. Host materials that are available in single-crystal form are preferred, either as bulk crystals or epitaxial layers; high structural quality is essential to suppress interactions with point defects or extended defects, or with paramagnetic impurities that could affect the defect spin state. The ability to easily process the host material (e.g., with standard wet etching techniques) is also an asset when considering future device development.

Defects with optical transitions in the near-infrared $(0.89-1.65 \mathrm{eV})$ or visible $(1.65-3.10 \mathrm{eV})$ regions of the spectrum are favored because of the ready availability of optical equipment compatible with these energies. The requirement that defect states lie well away from the band edges then points toward host materials that have sufficiently wide bandgaps. Such hosts will typically be fairly ionic and/or contain constituents taken from the first few rows of the periodic table. Diamond, with a bandgap of $5.5 \mathrm{eV}$, clearly fits; and staying with the Group IV elements, $\mathrm{SiC}$ is an obvious candidate. Among compounds, oxides tend to have large gaps and may satisfy a number of the other criteria such as zero nuclear spin. Nitrides such as GaN and AlN also have large bandgaps, and availability of these materials is rapidly increasing due to their use for solid-state lighting and power electronics. Nitrogen does not have zero nuclear spin, but this is not necessarily a strict requirement.

\section{Qubits in SiC}

Silicon carbide is closely related to diamond in structural and electronic properties. It is tetrahedrally coordinated, every $\mathrm{C}$ being surrounded by four $\mathrm{Si}$ atoms, and vice versa. A silicon vacancy in $\mathrm{SiC}$ is therefore surrounded by four $\mathrm{C}$ atoms and may be expected to exhibit behavior very similar to a vacancy in diamond. The most common polytypes, $4 \mathrm{H}-$ and $6 \mathrm{H}-\mathrm{SiC}$, have wide bandgaps of $3.27 \mathrm{eV}$ and $3.02 \mathrm{eV}$, respectively. ${ }^{21}$ The use of defects in $\mathrm{SiC}$ for spintronics was proposed by Gali in $2010,,^{22}$ and recent experiments have demonstrated that certain defects in $\mathrm{SiC}$ exhibit optically addressable spin states with long coherence times. ${ }^{23,24}$ Hybrid functional calculations for the Si vacancy and for an NV center in $4 \mathrm{H}-\mathrm{SiC}$ have indicated that these defects are indeed promising qubit candidates. ${ }^{16,25}$

\section{NV center in SiC}

Since a $\mathrm{Si}$ vacancy in $\mathrm{SiC}$ is expected to behave similarly to a vacancy in diamond, placing an $\mathrm{N}$ atom next to the $\mathrm{Si}$ vacancy should create a center similar to the NV center in diamond. To obtain a spin-triplet ground state, the $\left(\mathrm{N}_{\mathrm{C}}-\mathrm{V}_{\mathrm{Si}}\right)$ center should be negatively charged. ${ }^{16,25}$ Hybrid functional calculations have indicated that in $4 \mathrm{H}-\mathrm{SiC}$, this requires the Fermi level to lie between $1.60 \mathrm{eV}$ and $2.83 \mathrm{eV}$ above the valence band. Unlike the fortuitously favorable situation in diamond, judicious Fermi-level engineering (through controlled doping) is required here to achieve the desired charge state.

The calculated configuration coordinate diagram for the $\mathrm{NV}$ center in $4 \mathrm{H}-\mathrm{SiC}$ (from Reference 16) is shown in Figure 3. The optical transitions occur at about half the energy of those for the NV center in diamond; for instance, the zero-phonon line (ZPL) of the NV center in $4 \mathrm{H}-\mathrm{SiC}$ occurs at $1.09 \mathrm{eV}$, compared with $2.02 \mathrm{eV}$ for the $\mathrm{NV}$ in diamond. This difference is due to the larger lattice constant of $\mathrm{SiC}$ compared to diamond, which leads to a smaller overlap among the carbon $s p^{3}$ dangling-bond orbitals, and hence smaller splitting between the $a_{l}(2)$ and $e$ levels. This defect has not yet been observed experimentally.

\section{Silicon vacancy in SiC}

Silicon vacancies have also been detected in various polytypes of $\mathrm{SiC}$. Three zero-phonon lines in the photoluminescence spectrum of $6 \mathrm{H}-\mathrm{SiC}$, located in the near infrared between $1.35 \mathrm{eV}$ and $1.45 \mathrm{eV}$, have been attributed to silicon vacancies at the three inequivalent sites in $6 \mathrm{H}-\mathrm{SiC}^{26,27} \mathrm{~V}_{\mathrm{Si}}(\mathrm{h}), \mathrm{V}_{\mathrm{Si}}(\mathrm{k} 1)$, and $\mathrm{V}_{\mathrm{Si}}(\mathrm{k} 2)$; " $\mathrm{k}$ " and " $\mathrm{h}$ " indicate quasi-cubic and quasi-hexagonal sites, respectively. Various first-principles calculation ${ }^{25,28}$ have indicated that $\mathrm{V}_{\mathrm{Si}}$ incorporates in a high spin state in $3 \mathrm{C}$ - and $4 \mathrm{H}-\mathrm{SiC}$ and may act as a suitable qubit for quantum computing in these and other polytypes of SiC. Specifically, recent calculations ${ }^{25}$ show that $\mathrm{V}_{\mathrm{Si}^{-}}{ }^{-2}$ is stable in $n$-type $4 \mathrm{H}-\mathrm{SiC}$ and 
a

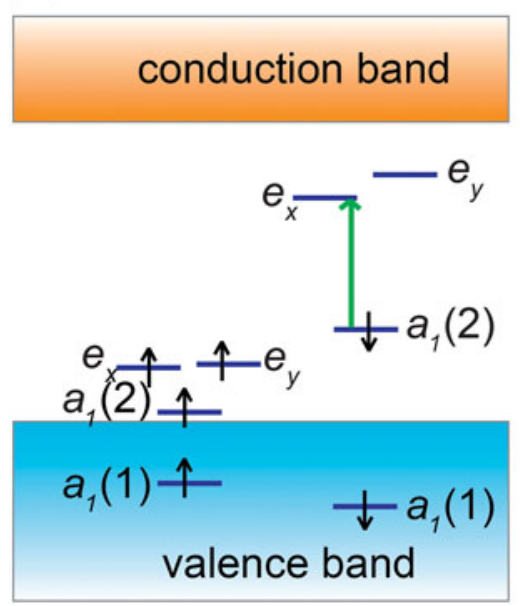

$\mathrm{b}$

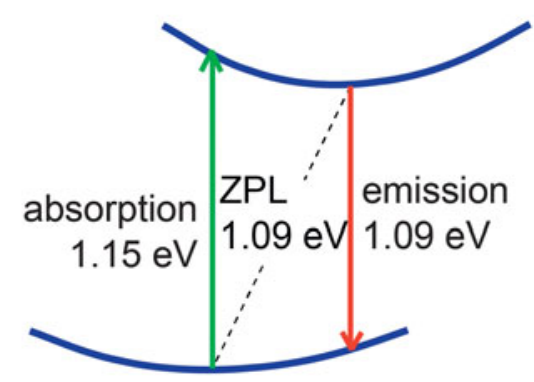

Figure 3. (a) Electronic structure of the negatively charged nitrogen-vacancy (NV) center $\left(\mathrm{NV}^{-1}\right)$ in $4 \mathrm{H}-\mathrm{SiC}$, as calculated with hybrid density functional theory. Adapted from Reference 16 . The positions of the defect states are qualitatively similar to those in the NV center in diamond (Figure 2a), but they are located closer to the band edges. Filling the electronic states in order of increasing energy leads to the occupation shown in the figure, resulting in a spin-1 (triplet) state for the center. (b) Calculated configuration coordinate diagram for the NV center in $4 \mathrm{H}-\mathrm{SiC}$. ZPL, zero-phonon line.

assumes a high spin state, due to the broken tetrahedral symmetry that splits the $t_{2}$ states. Calculations also indicate that the charge neutral $\mathrm{V}_{\mathrm{Si}}$ is a spin-triplet center, stable in $p$-type as well as in insulating $4 \mathrm{H}-\mathrm{SiC}$ (i.e., for Fermi level positions less than $1.4 \mathrm{eV}$ above the valence band) ${ }^{25}$ Optical excitation of $\mathrm{V}_{\mathrm{Si}}$ preferentially pumps the system into specific spin sublevels of the ground state, as experimentally demonstrated by Soltamov et al. ${ }^{29}$ for $4 \mathrm{H}-$ and $6 \mathrm{H}-\mathrm{SiC}$.

\section{Divacancy in SiC}

The first paramagnetic defect to be coherently manipulated in any polytype of $\mathrm{SiC}$ was the divacancy in $4 \mathrm{H}-\mathrm{SiC}{ }^{24}$ Photoluminescence spectra display a number of distinct zerophonon lines between $1.09 \mathrm{eV}$ and $1.20 \mathrm{eV}$. Photo-enhanced spin resonance and annealing experiments have indicated that the first four of these peaks, known by the singular label UD-2, can be attributed to the neutral divacancy. ${ }^{30,31}$ This is a charge-neutral defect complex consisting of a carbon vacancy adjacent to a silicon vacancy. Such a defect can assume four different configurations in $4 \mathrm{H}-\mathrm{SiC}$, as illustrated in Figure 4. These have been shown to form localized, paramagnetic electronic states that can be spin-polarized with incident light. ${ }^{24}$

The divacancy signals can be optically detected and coherently controlled. A pulse of light can polarize all the defect spins in the ensemble, and then the ensemble can be coherently manipulated with pulsed microwaves. Excitation with a second pulse of light and measurement of the photoluminescence intensity (a spin-dependent process) allow for a spindependent readout of the qubit state. This procedure can be applied to all four inequivalent forms of the divacancy. Very recently, it has been shown that analogous defect centers exist in $3 \mathrm{C}$ - and $6 \mathrm{H}-\mathrm{SiC}$, which can also be manipulated and optically measured. ${ }^{23}$ The microscopic nature of these defects is still unknown.

\section{Summary and outlook}

The outstanding properties of the NV center in diamond have motivated a concerted search for defect centers in other hosts that can similarly be used as qubits. We have outlined a set of criteria for defects and hosts, which can serve as a template for systematically investigating (or even designing) promising defect centers as candidate qubits. Two defects in $\mathrm{SiC}$ have been identified: the silicon vacancy, $\mathrm{V}_{\mathrm{Si}}$, and the $\mathrm{N}_{\mathrm{C}}-\mathrm{V}_{\mathrm{Si}}$ center. Experiments on optical initialization and control of divacancy centers have already demonstrated that $\mathrm{SiC}$ is a promising material.

Several new defect-based qubits have recently been computationally investigated: Chanier et al. ${ }^{32}$ have identified substitutional nickel impurities in diamond as potential qubits, and Yan et $a . .^{33}$ have proposed the neutral $\mathrm{V}_{\mathrm{Ga}}-\mathrm{O}_{\mathrm{N}}$ complex in zinc-blende GaN. A vast range of defect centers in other materials remains to be explored. Many of the materials that can host useful singlespin centers are already being produced with high quality

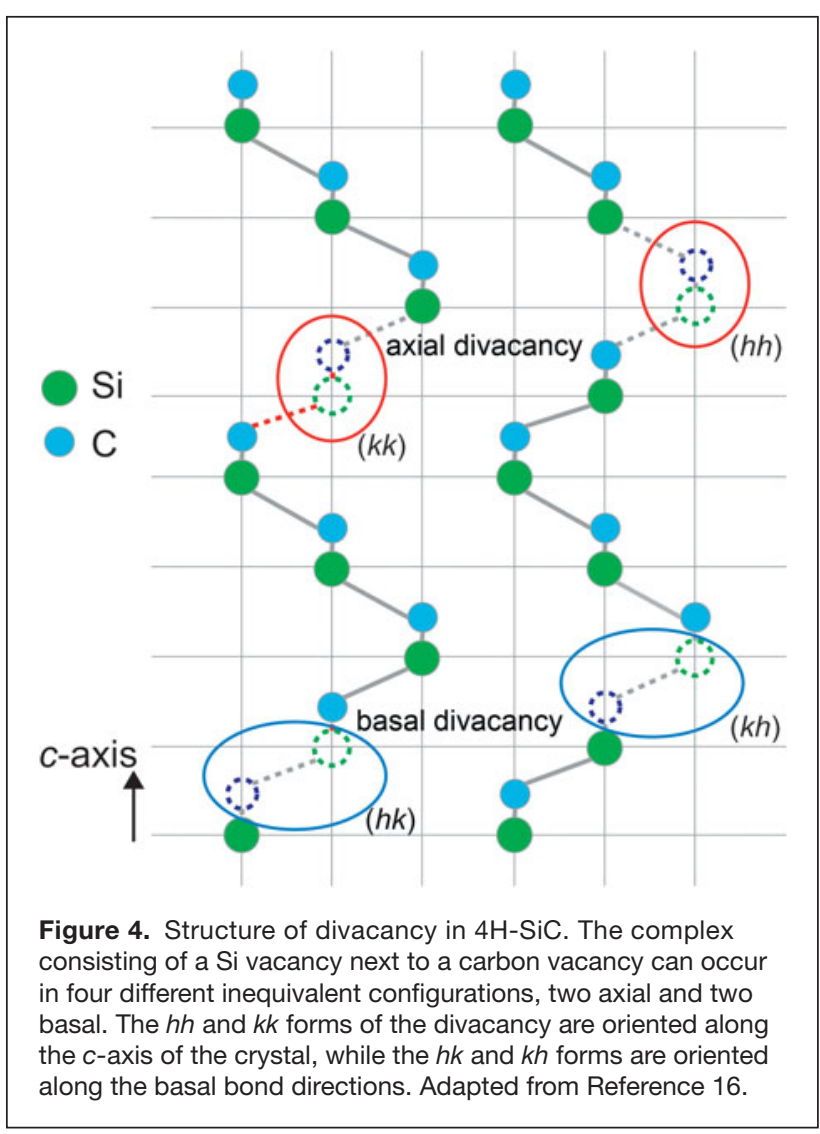


for other applications, but further advances in high-sensitivity characterization techniques will be required to speed up the search for potential qubits. Optical polarization has been reported for vacancy-related complexes in the cubic semiconductors $\mathrm{MgO}$ and $\mathrm{CaO},{ }^{34,35}$ which may herald a new path to defect-based qubits in octahedrally coordinated semiconductors. In addition, while the emphasis in the present article was on vacancy-related centers, other point defects (such as antisites) as well as impurities can be investigated in the same fashion. Chromium-implanted diamond leads to a fully polarized single-photon emitter with a sharp ZPL at $1.66 \mathrm{eV} ; 36$ however, the local structure of this defect is currently unknown. Substitutional transition metals may be a promising avenue for research since they tend to incorporate in high-spin states; in fact, a six-level system based on Mn has been realized in $\mathrm{MgO} .^{37}$ Investigations on these and other centers offer great potential for qubit functionalization, but will also deepen our understanding of and insight in this fascinating area of physics.

\section{Acknowledgments}

We are grateful to A. Alkauskas, B. Buckley, and W. Koehl for collaborations and discussions. This work was supported by the AFOSR MURI Program on Quantum Memories. Computational resources were provided by the Center for Scientific Computing at the CNSI and MRL (an NSF MRSEC, DMR-1121053) (NSF CNS-0960316), and by XSEDE (NSF OCI-1053575 and DMR070072N).

\section{References}

1. A.M. Childs, W. van Dam, Rev. Mod. Phys. 82, 1 (2010).

2. D.G. Cory, R. Laflamme, E. Knill, L. Viola, T.F. Havel, N. Boulant, G. Boutis, E. Fortunato, S. Lloyd, R. Martinez, C. Negrevergne, M. Pravia, Y. Sharf, G. Teklemariam, Y.S. Weinstein, W.H. Zurek, Fortschr. Phys. 48, 875 (2000).

3. C. Monroe, Nature 416, 238 (2002).

4. E. Lucero, R. Barends, Y. Chen, J. Kelly, M. Mariantoni, A. Megrant, P. O’Malley, D. Sank, A. Vainsencher, J. Wenner, T. White, Y. Yin, A.N. Cleland, J.M. Martinis, Nat. Phys. 8, 719 (2012).

5. R. Hanson, D.D. Awschalom, Nature 453, 1043 (2008).

6. M. McCluskey, E. Haller, Dopants and Defects in Semiconductors (CRC Press, FL, 2012).

7. M. Stavola, Identification of Defects in Semiconductors and Semimetals (Academic, San Diego, 1999), Vols. 51A, 51B.

8. G. Davies, M.F. Hamer, Proc. R. Soc. London, Ser. A 348, 285 (1976).

9. P. Hohenberg, W. Kohn, Phys. Rev. 136, B864 (1964).

10. W. Kohn, L.J. Sham, Phys. Rev. 140, A1133 (1965).

11. C.G. Van de Walle, J. Neugebauer, J. Appl. Phys. 95, 3851 (2004)

12. J. Heyd, G.E. Scuseria, M. Ernzerhof, J. Chem. Phys. 118, 8207 (2003); J. Chem. Phys. 124, 219906(2006).

13. C.G. Van de Walle, A. Janotti, Phys. Status Solidi B 248, 19 (2011).

14. A. Gali, E. Janzén, P. Deak, G. Kresse, E. Kaxiras, Phys. Rev. Lett. 103, 186404 (2009).

15. N.B. Manson, J.P. Harrison, M.J. Sellars, Phys. Rev. B. 74, 104303 (2006). 16. J.R. Weber, W.F. Koehl, J.B. Varley, A. Janotti, B.B. Buckley, C.G. Van de Walle, D.D. Awschalom, Proc. Natl. Acad. Sci. U.S.A. 107, 8513 (2010).

17. V. Acosta, P. Hemmer, MRS Bull. 38, 127 (2013).

18. D. Toyli, D.J. Christie, A. Alkauskas, B.B. Buckley, C.G. Van de Walle, D.D. Awschalom, Phys. Rev. X2, 031001 (2012).

19. N. Bar-Gill, L.M. Pham, A. Jarmola, D. Budker, R.L. Walsworth, Nat. Commun. 4, 1743 (2013).

20. G.D. Fuchs, V.V. Dobrovitski, D.M. Toyli, F.J. Heremans, D.D. Awschalom, Science 326, 1520 (2009).

21. 0. Madelung, Semiconductors: Data Handbook (Springer-Verlag, NY, 2004).

22. A. Gali, A. Gällström, N.T. Son, E. Janzén, Mater. Sci. Forum 645, 395 (2010).
23. A.L. Falk, B.B. Buckley, G. Calusine, W.F. Koehl, V.V. Dobrovitski, A. Politi, C.A. Zorman, P.X.-L. Feng, D.D. Awschalom, Nat. Commun. 4, 1819 (2013).

24. W.F. Koehl, B.B. Buckley, F.J. Heremans, G. Calusine, D.D. Awschalom, Nature 479, 84 (2011).

25. J.R. Weber, W.F. Koehl, J.B. Varley, A. Janotti, B.B. Buckley, C.G. Van de Walle, D.D. Awschalom, J. Appl. Phys. 109, 102417 (2011).

26. M. Wagner, B. Magnusson, W.M. Chen, E. Janźen, E. Söman, C. Hallin, J.L. Lindström, Phys. Rev. B. 62, 16555 (2000).

27. S.B. Orlinski, J. Schmidt, E.N. Mokhov, P.G. Baranov, Phys. Rev. B. 67, 125207 (2003).

28. L. Torpo, R.M. Nieminen, K.E. Laasonen, S. Pöykkö; Appl. Phys. Lett. 74, 221 (1999).

29. V.A. Soltamov, A.A. Soltamova, P.G. Baranov, I.I. Proskuryakov, Phys. Rev. Lett. 108, 226402 (2012).

30. A. Gali, Phys. Status Solidi B 248, 1337 (2011).

31. N.T. Son, P. Carlsson, J. ul Hassan, E. Janzén, T. Umeda, J. Isoya, A. Gali, M. Bockstedte, N. Morishita, T. Ohshima, H. Itoh, Phys. Rev. Lett. 96, 055501 (2006).

32. T. Chanier, C.E. Pryor, M.E. Flatté; Europhys. Lett. 99, 67006 (2012).

33. X. Wang, M. Zhao, Z. Wang, X. He, Y. Xi, S. Yan, Appl. Phys. Lett. 100, 192401 (2012).

34. D.H. Tanimoro, W.M. Ziniker, J.O. Kemp, Phys. Rev. Lett. 14, 645 (1965).

35. B. Henderson, J. Phys. C 9, 2185 (1976).

36. I. Aharonovich, S. Castelletto, B.C. Johnson, J.C. McCallum, D.A. Simpson, A.D. Greentree, S. Prawer, Phys. Rev. B 81, 121201 (2010).

37. S. Bertaina, L. Chen, N. Groll, J. Van Tol, N.S. Dalal, I. Chiorescu, Phys. Rev. Lett. 102, 050501 (2009).

38. P.G. Neudeck, J. Electron. Mater. 24, 4 (1995).

39. V. Srikant, D.B. Clarke, J. Appl. Phys. 83, 10 (1998).

40. N. Kumbhojkar, V.V. Nikesh, A. Khsirsager, S. Mahamuni, J. Appl. Phys. 88, 6620 (2000).

41. E. Silveira, J.A. Freitas, O.J. Glembocki, G.A. Stack, L.J. Schowalter, Phys Rev. B 71, 041201 (2005).

42. P. Lawaetz, Phys. Rev. B 4, 3460 (1971).
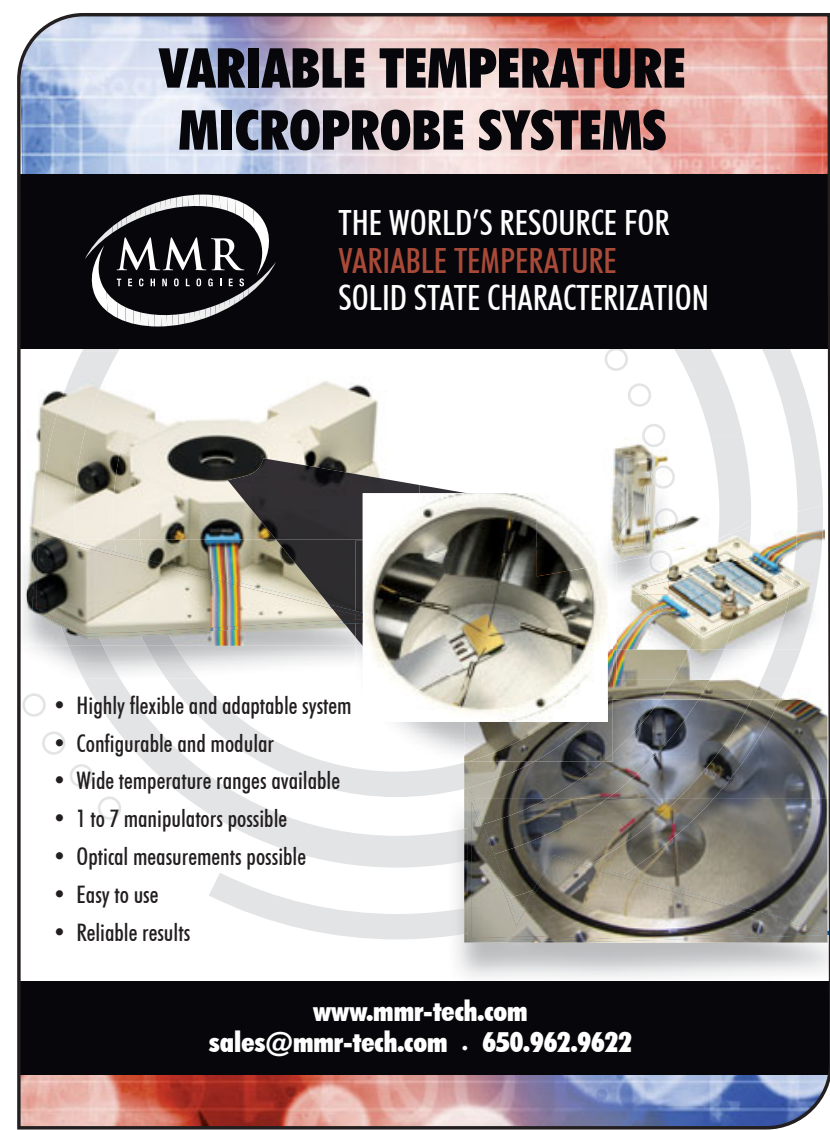


\section{Manipulation and}

Heating Stages

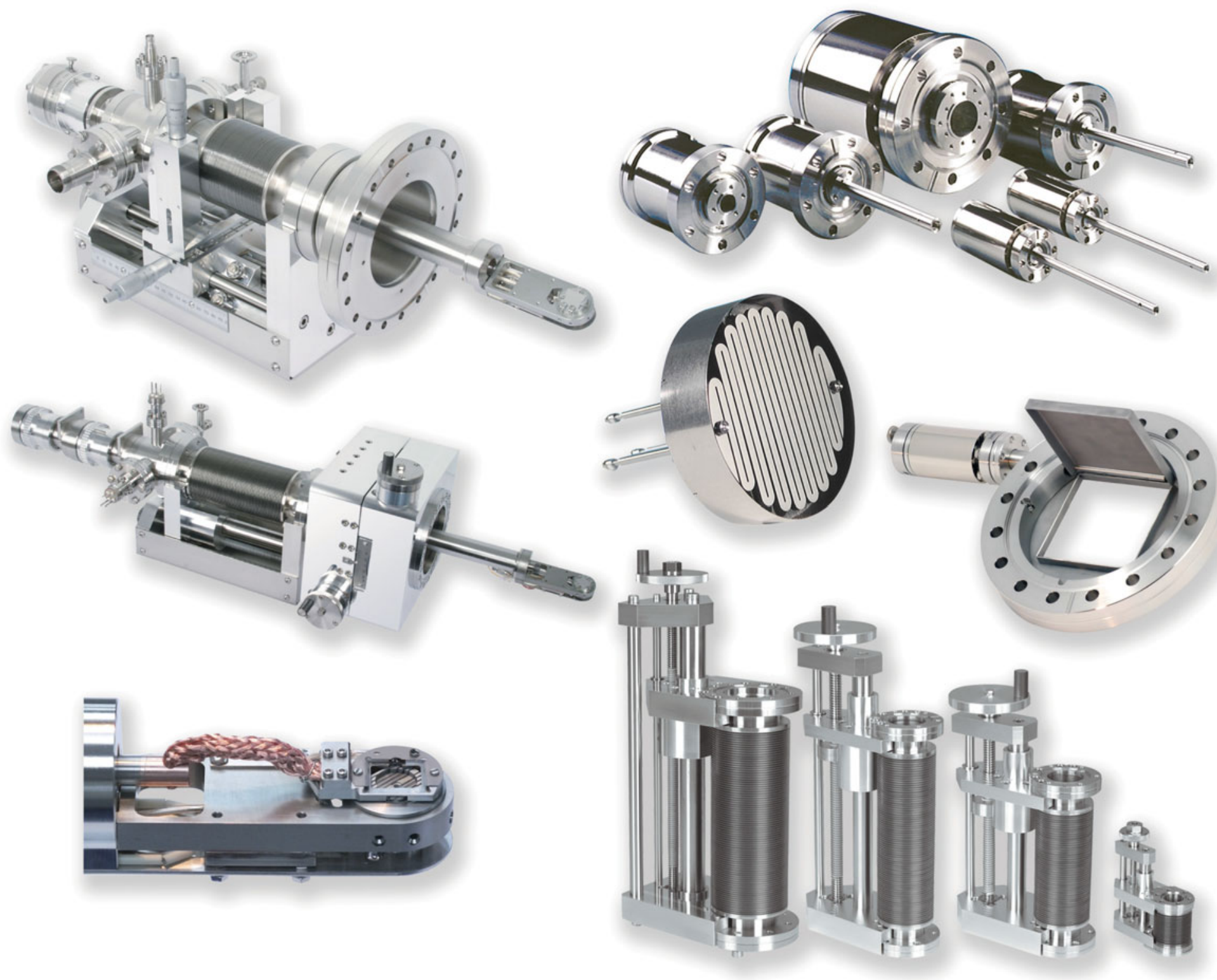

\section{Kurt J. Lesker"}

UH $\forall$ Design UHV MOTION AND HEATING 